\title{
CONCEPTUAL DESIGN OF ILC DAMPING RING WIGGLER STRAIGHT VACUUM SYSTEM*
}

\author{
S. Marks, K. Kennedy, D. Plate, R. D. Schlueter, M. Zisman, LBNL, Berkeley, CA 94720, U.S.A.
}

\section{Abstract}

The positron and electron damping rings for the International Linear Collider will contain long straight sections consisting of twenty wiggler/quadrupole pairs. The wigglers will be based upon the CESR superconducting design. There are a number of challenges associated with the design of the wiggler straight vacuum system, in particular, the absorption of photon power generated by the wigglers. This paper will present the overall conceptual design of the wiggler straight vacuum system developed for the ILC Reference Design Report. Particular emphasis will be placed on photon power load calculations and the absorber design.

\section{INTRODUCTION}

The magnet lattice adopted for the International Linear Collider (ILC) positron and electron damping rings for the Reference Design Report (RDR) contains a total of eighty damping wigglers per ring. The wigglers are incorporated into four straight sections, which incorporate 21 quadrupoles and twenty wigglers centered between quadrupole pairs. The wigglers are based upon the CESR superconducting design [1]. The primary technical challenges for the wiggler straight vacuum system consist of dealing with the substantial power eradiated from the wiggler and minimizing the secondary electron yield (SEY) in order to meet emittance objectives for the positron ring. The former challenge was the primary focus of technical work on the vacuum system within the RDR phase. Techniques for SEY reduction provide a significant focus for current and future work.

Table 1 summarizes the parameters relevant for the wiggler straight layout and radiated power load calculations.

Table 1: Wiggler Straight Parameters

\begin{tabular}{|l|l|}
\hline Total Length of Straight $(\mathrm{m})$ & 75 \\
\hline Quadrupole Spacing $(\mathrm{m})$ & 3.75 \\
\hline Wiggler Length (m) & 2.45 \\
\hline Wiggler Period (m) & 0.4 \\
\hline Wiggler Field Strength (T) & 1.67 \\
\hline Number of Full Strength Poles & 12 \\
\hline Electron Energy (GeV) & 5.0 \\
\hline Current (A) & 0.4 \\
\hline
\end{tabular}

*Work supported by the Office of Science, U. S. Department of Energy, under Contract No. DE-AC02-05CH112231
The first section of this paper presents a summary of the vacuum system conceptual design. The second section presents photon power load calculations and discuss the absorber design.

\section{VACUUM SYSTEM CONCEPT}

The wiggler straight vacuum system for the ILC damping rings consists of separate chambers for the wiggler and quadrupole sections. Fig. 1 shows a plan view layout of a portion of the wiggler straight.

A cross-section of the wiggler chamber is shown in Fig. 2. The overall width and height of the chamber indicated in the figure represent dimensions compatible with the proposed wiggler and cryostat design. The chamber is a machined and welded aluminum unit, designed as a warm-bore insert, mechanically decoupled from the wiggler and cryogenic system. A NEG pumping system and photon absorber are incorporated in antechambers. Integral cooling is incorporated to minimize distortion of the chamber and thermal load on the wiggler cryostat during NEG regeneration. A TiZrV NEG surface coating will be used on the main chamber bore to minimize SEY [2]. Note that the eventual vacuum system design may utilized other surface coatings such as TiN as well as incorporate other technologies, such as clearing electrodes, to minimize SEY.

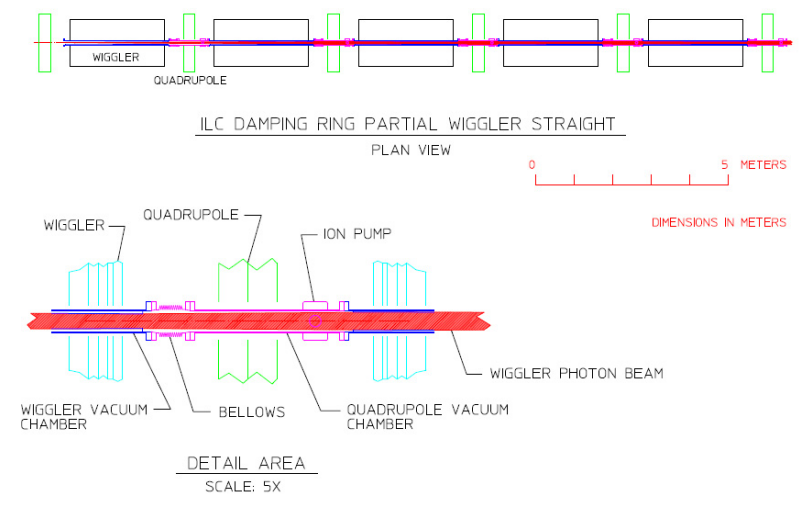

Figure 1. ILC wiggler straight layout.

The NEG pumping system will be similar to that designed for the PEP-II $B$ Factory [3]. The assembly consists of NEG coated fins and an integral heating rod for regeneration. The estimated pumping speed for $\mathrm{CO}$ is 1000 liters $/ \mathrm{sec} / \mathrm{m}$. With a total incident photon flux of $2 \times 10^{18}$ photons $/ \mathrm{s} / \mathrm{m}$, the estimated yield of $\mathrm{CO}$ will be $2 \times 10^{13} \mathrm{molecules} / \mathrm{s} / \mathrm{m}$. This will result in an equilibrium CO partial pressure of $7 \times 10^{-10}$ Torr. 


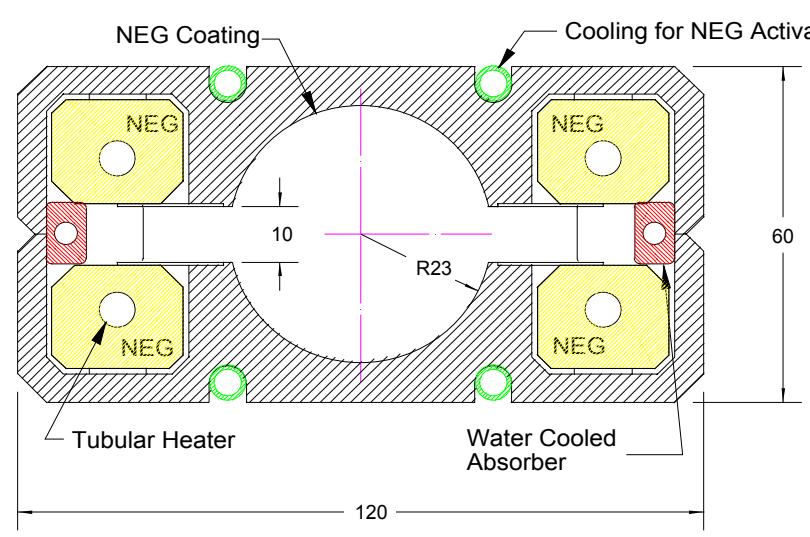

Figure 2. ILC damping ring wiggler chamber.

Between each wiggler chamber will be a separate chamber for the quadrupole. The cross section is shown in Fig. 3. This chamber is welded aluminum, also incorporating TiZrV NEG coating for SEY reduction. Bellows, a BPM assembly and an ion pump, for pumping non-reactive gases, are incorporated. The ion pump also doubles as a vacuum gauge. The quadrupole chamber is completely shadowed by the wiggler chamber photon absorbers and does not absorb any of the direct photon power from upstream wigglers.

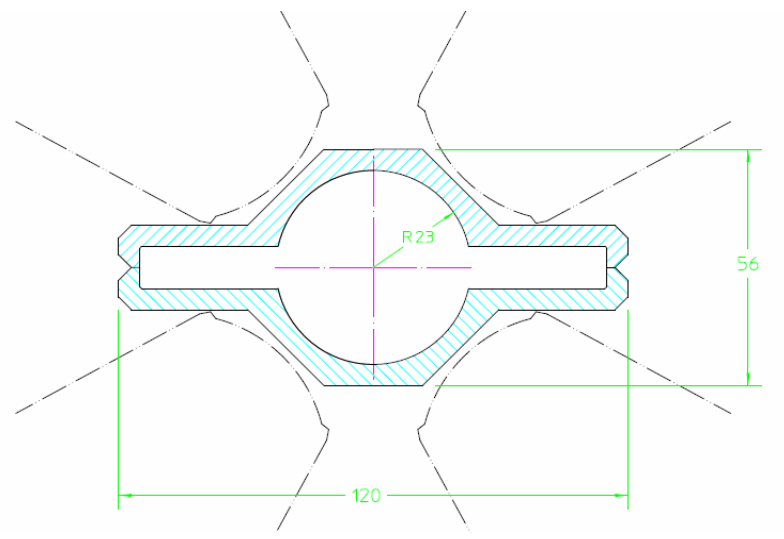

Figure 3. Cross section of quadrupole chamber.

\section{PHOTON POWER ABSORPTION}

Each wiggler emits a total of $17 \mathrm{~kW}$ over a fan of \pm 6.4 mrad. Due to the length of the straight, a significant portion of the total $340 \mathrm{~kW}$ emitted must be absorbed within the straight. We can consider the following two options for photon absorption:

1. Absorb power within the quadrupole section via a mask that shadows successive wiggler chambers.

2. Absorb power within the wiggler sections.

Option 1 was initially preferred, but, as discussed below, proved to be impractical.

The following assumptions were made to arrive at an absorber design:

1. The maximum allowable surface power density is $15 \mathrm{~W} / \mathrm{mm}^{2}$.
2. The minimum allowable absorber slope is $1 / 10$.

3. Allow for a $\pm 10 \mathrm{~mm}$ horizontal and a $\pm 5 \mathrm{~mm}$ vertical beam offset.

4. Transitions between different apertures must be tapered with a $1 / 10$ slope.

Assumption 1 is based upon the use of copper as the absorbing material and is consistent with standard design practice for synchrotron light source photon absorbers. For example the peak power density for blank-off flanges in the ALS storage ring is $11.5 \mathrm{~W} / \mathrm{mm}^{2}$ [4]. In recent designs developed for front end components at ESRF peak power densities have been pushed to over $20 \mathrm{~W} / \mathrm{mm}^{2}$ [5]. However, these are very aggressive designs developed out of necessity. Our assumtion is a reasonable initial limit.

Assumption 2 is based upon consideration for reasonably achievable tolerances. Although a beam missteer budget has not been formally established, assumption 3 is considered conservative yet reasonable. Assumption 4 is consistent with standard practice to prevent trapped RF modes.

Photon power densities are calculated with the program $W r P w r$ [6], which calculates angular power densities for rays hitting a specified target. For each ray, angular power density is converted to area power density based upon the target surface orientation and distance from the source. The source is defined in terms of a periodic magnetic field, with the number of full strength peaks and field harmonics specified.

\section{Photon Mask Within Quadrupole Section}

The objective of placing a photon absorber within the quadrupole section is to completely mask the wiggler chambers. The required horizontal mask aperture is constrained by the minimum electron/positron beam stay clear and the maximum allowable power density on the mask. We assume a minimum horizontal beam stay clear of $25 \mathrm{~mm}$. We also assume, as a starting point, that the horizontal aperture for the wiggler chamber is $45 \mathrm{~mm}$, as is the case for the existing CESR wiggler chamber.

For the assumed horizontal apertures, and with no beam mis-steer, photons from the first wiggler will be intercepted by a mask between wigglers two and three. If there is a $10 \mathrm{~mm}$ horizontal beam offset, photons will be absorbed between the first and second wigglers. The highest power density occurs at the end of the stratight between wigglers nineteen and twenty. The peak normal power density is $1170 \mathrm{~W} / \mathrm{mm}^{2}$ without mis-steer and 1900 $\mathrm{W} / \mathrm{mm}^{2}$ for a $10 \mathrm{~mm}$ beam offset. These power densities will be reduced by a factor of 10 for our assumed slope. This results in a surface power density that is still an order of magnitude above our design maximum. Alternatively, if we set the slope at 0.008 , the peak power density will be $15 \mathrm{~W} / \mathrm{mm}^{2}$. However, this requires an absorber length of $2.5 \mathrm{~m}$, which is not compatible with the space allotted for the quadrupoles, and represents an impractical placement tolerance to achieve this slope relative to the beam. 
The only way to decrease the peak power density at the absorber while maintaining a reasonable slope is to increase the horizontal aperture of the mask, and thus the chamber correspondingly to maintain shadowing by the mask. Fig. 3 is a plot of peak power density as a function of horizontal mask aperture. Notice that a mask aperture of $150 \mathrm{~mm}$, and the corresponding wiggler chamber aperture of $170 \mathrm{~mm}$, are required to achieve an acceptable power density. This would require a major redesign of the wiggler and cryostat.

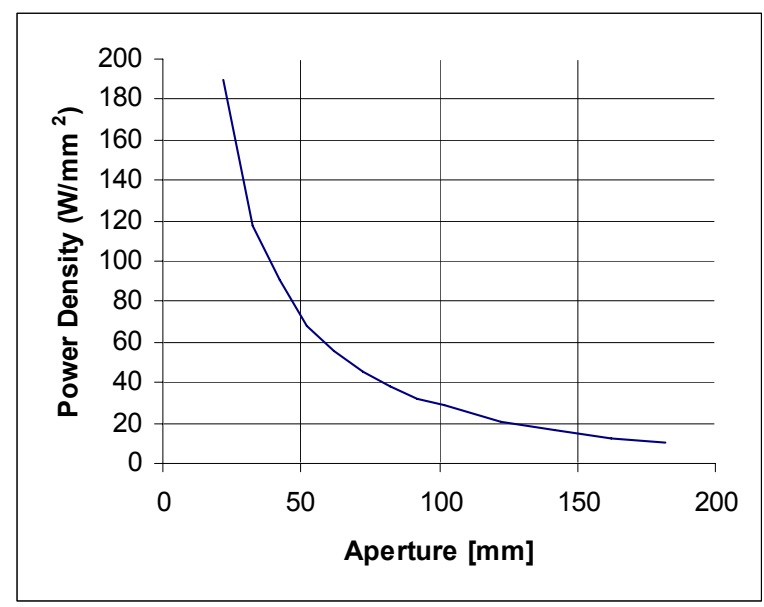

Figure 4. Peak power density on photon mask, with a $1 / 10$ absorber slope, as a function of mask horizontal aperture.

\section{Photon Absorber Within the Wiggler Chamber}

Due to the difficulty in absorbing photon power outside of the wiggler chambers, as detailed above, an alternative design for absorbers incorporated within the wiggler chambers was developed. The photon absorbers are hollow water-cooled copper conductor. The horizontal placement of the absorber surface is $48 \mathrm{~mm}$ at the chamber entrance, and tapers to $38 \mathrm{~mm}$ at the exit in order to shadow the downstream quadrupole chamber. Power radiated from the first wiggler in the straight is intercepted first by the absorber at wiggler number three. Intercepted power increases for successive wigglers up to number nine, where a constant $3 \mathrm{~W} / \mathrm{mm}^{2}$ peak power density is achieved for wigglers nine and beyond. Figure 4 shows calculated power density distribution on an absorber. The total power absorbed per wiggler is $26 \mathrm{~kW}, 13 \mathrm{~kW}$ per absorber. The peak power density and total power loads on the absorbers are relatively modest and consistent with the use of standard hollow water cooled copper.

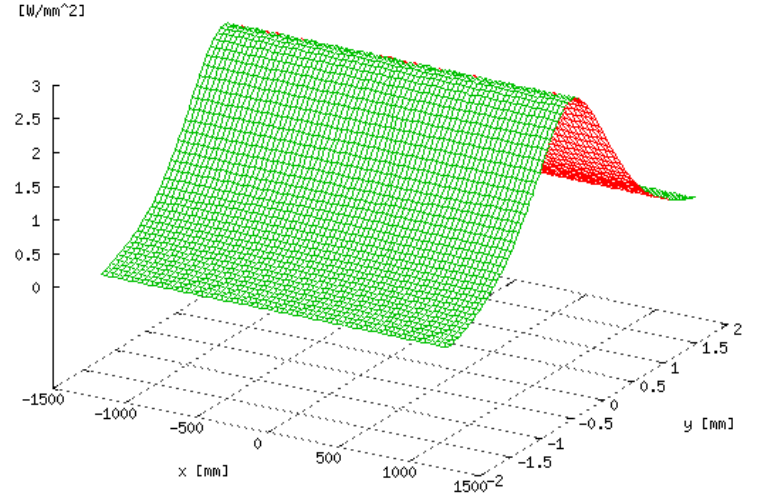

Figure 5. Power density distribution on absorber.

\section{CONCLUSION}

A conceptual design of the ILC damping ring wiggler straight vacuum system has been developed for the RDR phase. The NEG pumping system, augmented by ion pumps, will provide adequate pumping. The initial concept incorporates a NEG coating within the beam chamber to minimize SEY. Pending the results of further studies, other technologies may be utilized for SEY minimization. A photon absorber integral to the wiggler chamber has been developed. The peak power densities and total power loads are relatively modest.

\section{REFERENCES}

[1] J. A. Crittenden, et al., "Design Considerations for the CESR-c Wiggler Magnets", Proceedings of the 2003 Particle Accelerator Conference, Volume 3, Issue , 12-16 May 2003 Page(s): 1954 - 1956 vol.3.

[2] F. Le Pimpac, et al., "Secondary Electron Yield Measurements of TiN Coating and TiZrV Getter Film", SLAC-TN-03-052, August, 2004.

[3] L. Bertolini, et al., "Design of the Linear NonEvaporable Getter Pump for the PEP-II B Factory", Proceedings of 1997 Particle Accelerator Conference, Volume 3, Issue , 12-16 May 1997 Page(s):3622 - 3624 vol. 3

[4] C. A. Corradi, "Superbend - Photon Stops/Blank Flanges, Thermal and Structural Analysis", LBNL Engineering Note M7823A.

[5] P. Marion, et al., "New High Heat Load Beamline Components for the ESRF", Proceedings of $2^{\text {nd }}$ International Workshop on Mechanical Engineeringof Synchrotron Radiation Equipment and Instrumentation (MEDSIO2), http://www.aps.anl.gov/asd/me/medsi02.

[6] S. Marks, C. Cork, "Flux, Irradiance, and Transmission Calculations for the ALS Wiggler Beamline 5.0", Review of Scientific Instruments, Volume 67, Issue 9, September 1996, p.3377 